Anna Miller

\title{
In der digitalen Dauerschleife
}

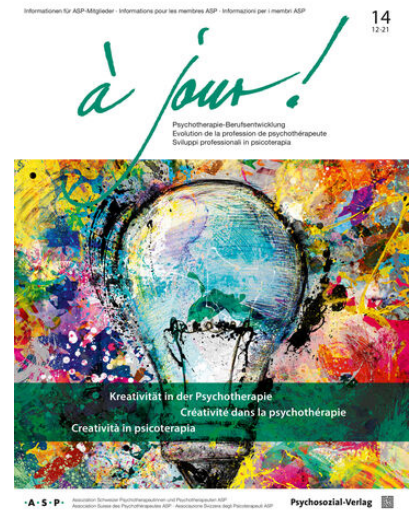

à jour!

Psychotherapie-Berufsentwicklung

7. Jahrgang, Nr. 2, 2021, Seite 33-34

DOI: 10.30820/2504-5199-2021-2-33

Psychosozial-Verlag 


\section{Impressum | Mentions légales}

\author{
à jour! - Psychotherapie-Berufsentwicklung \\ ISSN 2504-5199 (Print-Version) \\ ISSN 2504-5202 (digitale Version) \\ 7. Jahrgang Heft 2/2021, Nr. 14 \\ https://doi.org/10.30820/2504-5199-2021-2
}

\section{Herausgeber}

Assoziation Schweizer Psychotherapeutinnen und Psychotherapeuten ASP

Riedtlistr. 8 | 8006 Zürich | Tel.: 0432689300 | www. psychotherapie.ch

Die Zeitschrift à jour! Psychotherapie-Berufsentwicklung ist ein Informationsorgan der ASP. Gleichzeitig versteht sie sich als Forum ihrer Mitglieder, in dem auch Meinungen geäussert werden, die unabhängig von der Meinung des Vorstandes und der Redaktion sind.

\section{Redaktion}

Peter Schulthess | Redaktionsleitung peter.schulthess@psychotherapie.ch|Tel.: 0765591920

Marianne Roth | marianne.roth@psychotherapie.ch

Veronica Defiébre | veronica.defiebre@psychotherapie.ch

Sandra Feroleto|sandra.feroleto@psychotherapie.ch

\section{Redaktionsschluss}

1. März für Juni-Heft / 15. September für Dezember-Heft

\section{Verlag}

Psychosozial-Verlag

Walltorstr. 10 | D-35390 Gießen | Tel.: +4964196997826

www.psychosozial-verlag.de |info@psychosozial-verlag.de

\section{Abo-Verwaltung | Bezugsgebühren}

Psychosozial-Verlag | bestellung@psychosozial-verlag.de

Jahresabonnement 29,90€ (zzgl. Versand)

Einzelheft 19,90€ (zzgl. Versand)

Studierende erhalten gegen Nachweis $25 \%$ Rabatt.

Das Abonnement verlängert sich um jeweils ein Jahr, sofern nicht eine Abbestellung

bis acht Wochen vor Ende des Bezugszeitrasums erfolgt.

Das Abonnement ist für ASP-Mitglieder im Mitgliedsbeitrag enthalten.

\section{Anzeigen}

Anfragen zu Anzeigen richten Sie bitte an den Verlag (anzeigen@psychosozial-verlag.de) oder die Geschäftsstelle der ASP (asp@psychotherapie.ch).

Es gelten die Preise der auf www. psychosozial-verlag.de einsehbaren Mediadaten. ASP-Mitglieder wenden sich bitte direkt an die Geschäftsstelle der ASP.

Bildnachweise

Titelbild: @ iStock by Getty Images/RapidEye (ID 912441162)

\section{Digitale Version}

Die Zeitschrift à jour! Psychotherapie-Berufsentwicklung ist auch online einsehbar: www.a-jour-asp.ch

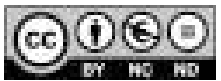

Die Beiträge dieser Zeitschrift sind unter der Creative Commons Attribution-NonCommercial-NoDerivs 3.0 DE Lizenz lizensiert. Diese Lizenz erlaubt die private Nutzung und unveränderte Weitergabe, verbietet jedoch die Bearbeitung und kommerzielle Nutzung. Weitere Informationen finden Sie unter: creativecommons.org/licenses/by-nc-nd/3.0/de

\author{
à jour! - Evolution de la profession de psychothérapeute \\ ISSN 2504-5199 (Version papier) \\ ISSN 2504-5202 (Version numérique) \\ 7. tome numéro 2/2021, 14 \\ https://doi.org/10.30820/2504-5199-2021-2
}

\section{Editeur}

Association Suisse des Psychothérapeutes ASP

Riedtlistr. 8 | 8006 Zürich | Tel.: 0432689300 | www. psychotherapie.ch

La revue à jour! Évolution de la profession du psychothérapeute est un organe d'information de l'ASP. En plus, c'est un forum dans lequel on exprime des avis qui sont indépendants de l'avis du comité et de la rédacion.

\section{Rédaction}

Peter Schulthess | Directeur de rédacion

peter.schulthess@psychotherapie.ch | Tel.: 0765591920

Marianne Roth / marianne.roth@psychotherapie.ch

Veronica Defiébre /veronica.defiebre@psychotherapie.ch

Sandra Feroleto|sandra.feroleto@psychotherapie.ch

\section{Date de rédaction finale}

1er mars pour juin | 15 septembre pour décembre

\section{L'éditeur}

Psychosozial-Verlag

Walltorstr. 10 | D-35390 Gießen | Tel.: +49641969978 26

www.psychosozial-verlag.de|info@psychosozial-verlag.de

\section{Gestion des abonnements | Frais de souscription}

Édition psychosociale | bestellung@psychosozial-verlag.de

Abonnement annuel 29,90€ (frais d'envoi en sus)

Prix du numéro 19,90€ (frais d'envoi en sus)

Les étudiants bénéficient d'une réduction de $25 \%$ sur présentation d'un justificatif.

L'abonnement est reconduit d'un an à chaque fois dans la mesure où aucune résiliation n'a lieu avant le 15 novembre.

L'abonnement est compris dans la cotisation pour les membres ASP.

\section{Annonces}

Veuillez adresser vos demandes de renseignements sur les annonces à l'éditeur (anzeigen@psychosozial-verlag.de) ou au bureau de I'ASP (asp@psychotherapie.ch). Les prix valables sont ceux publiés dans les données médiatiques sur www.psychosozial-verlag.de.

Les membres ASP sont priés de s'adresser directement à la rédaction.

\section{Crédits photographiques}

Couverture: $\odot$ iStock by Getty Images/RapidEye (ID 912441162)

\section{Version numérique}

La revue à jour! Psychothérapie-Développement professionnel est également consultable en ligne : www. a-jour-asp.ch

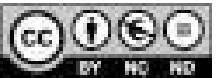

Les articles de cette revue sont disponibles sous la licence Creative Commons 3.0 DE en respectant la paternité des contenus - pas d'utilisation commerciale - sans œuvre dérivée. Cette licence autorise l'utilisation privée et la transmission sans modification, interdit cependant le traitement et l'utilisation commerciale. Veuillez trouver de plus amples informations sous : creativecommons.org/licenses/by-nc-nd/3.0/de 


\section{In der digitalen Dauerschleife}

Anna Miller

Sprechen wir über psychische Gesundheit, müssen wir auch über unseren Umgang mit dem Digitalen sprechen. Und in eine digitale Balance zu finden. Als Fachpersonen genauso wie privat.

Wann haben Sie das letzte Mal einfach in die Weite geschaut? Wann haben Sie für Minuten, vielleicht sogar für Stunden einmal Ihr digitales Leben vergessen, haben das Handy weggelegt und Ihren Gedanken gelauscht, ohne etwas zu tun, etwas nachzuschauen oder abzuarbeiten? 2007 läutete ein hagerer Mann in einem schwarzen Rollkragenpullover eine neue Ära ein. Nicht einmal 15 Jahre später betreten wir im Alltag ständig Räume, in denen Menschen zwar noch physisch da sind, aber geistig und emotional weit weg. Wir leben in einer Welt, in der in China Internet-Entwöhnungs-Lager für Jugendliche stattfinden, in der 14-jährige Mädchen eine Essstörung entwickeln, weil ihr Spiegelbild nicht mehr kohärent ist mit dem Foto, das sie für Instagram durch einen Filter gejagt haben. Dem Durchschnitt der Gesellschaft gelingt es heute

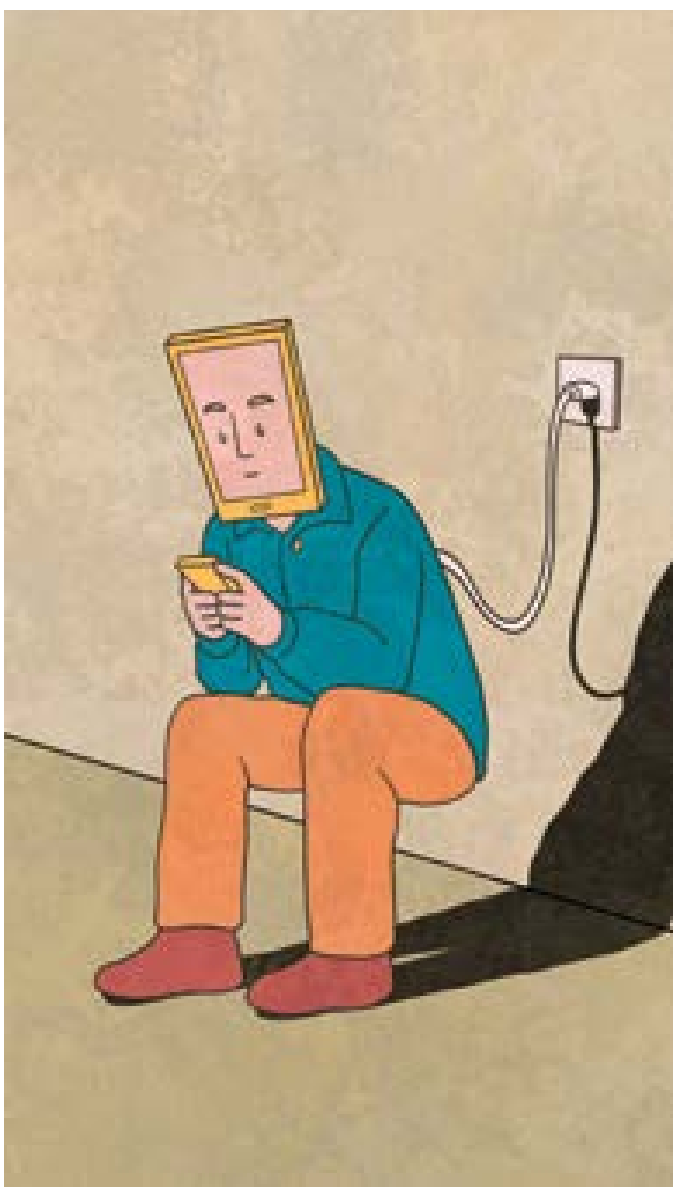

nicht mehr länger als zehn Minuten, sich auf etwas zu konzentrieren. Schlafstörungen haben zugenommen, genauso wie Angststörungen und depressive Verstimmung. Das hat verschiedenste Gründe. Und doch ist einer davon in der Dauerpräsenz des Digitalen zu suchen.

Wir leben in einer always on-Gesellschaft, sind immer erreichbar, immer öfter an den Geräten. Nicht nur bei der Arbeit oder wenn wir Pizza bestellen, sondern auch in Bereichen, die der Erholung und der menschlichen Verbundenheit dienen: beim Spaziergang im Wald, bei einem Essen mit Freunden, im Bett mit Partner oder Partnerin. Und die Pandemie hat die Zeit, die wir vor Bildschirmen verbringen, noch einmal massiv verlängert. Doch die Bildschirmzeit allein, das zeigen Studien, ist nicht das ganze Problem.

Denn man kann vor dem Bildschirm aktiv sein, kann etwas erschaffen, zum Beispiel, wenn man einen Dokumentarfilm schneidet. Das grundlegende Problem bei der Bildschirmdauer ist das passive Konsumieren von teils destabilisierenden Inhalten wie News oder das vermeintlich perfekte Leben der anderen, und, dass wir vor dem Bildschirm sitzend nichts mehr real erleben. Der Mensch, das weiss die Positive Psychologie, braucht Bewegung, echte Berührung, Natur, Sexualität und kreative Entfaltung, um glücklich zu sein.

Und genau hier liegt das Problem der aktuellen Debatte: Denken wir an das Wort Digitalisierung, dreht sich im öffentlichen Diskurs oft noch alles um technologische Aspekte: E-ID, selbstfahrende Autos, Digitalisierung von Bibliotheksbeständen. Wir reden von 5G-Antennen und davon, welche Berufe sich wie verändern oder ganz verschwinden. Wenn wir aber vom psychologischen Standpunkt aus an das Wort Digitalisierung herantreten, berühren wir automatisch die Frage, was sie mit dem Menschen und seiner Psyche macht. Mit der Art, wie er lebt, arbeitet, in Beziehung tritt. Und was ein Leben vor dem Bildschirm mit ihm macht.

Dabei sind wir nicht nur immer länger und passiver online, sondern betreiben auch das sogenannte compulsive checking - die ständige Unterbrechung von dem, was wir tun, um das Smartphone zu bedienen. Dabei sind wir getrieben von Hormonen: Das Dopamin, das ausgeschüttet wird, wenn wir eine Nachricht erhalten oder einen Like, lässt uns kurzzeitig zufrieden

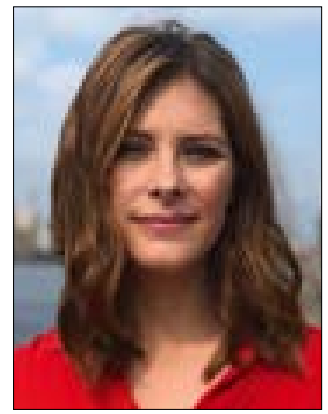


sein, das Cortisol, das ausgeschüttet wird, wenn wir mal nicht sofort nachschauen, drängt uns dazu, das Verhalten, das schon lange Suchttendenzen aufweist, möglichst rasch $\mathrm{zu}$ wiederholen, um das System zu beruhigen - vor allem in Form eines Überkonsums von Kommunikationsapplikationen. Weil Verhaltenspsychologen und grössenwahnsinnige CEOs einiger Silicon Valley-Firmen lange daran gearbeitet haben, den Menschen dort zu triggern, wo er am verwundbarsten ist: in seinem Bedürfnis nach Verbindung und Zugehörigkeit.

Am Ende leben wir damit in einem Zustand, der von digitalen Impulshandlungen dominiert wird. Der Auswirkungen hat auf unsere Fähigkeit, Wissen aufzunehmen, in Beziehung zu treten, überhaupt in einen kreativen Flowzustand zu kommen. Diese Art von digitaler Interaktion schadet uns langfristig auf neurobiologischer Ebene. Sie lässt unseren Körper in einem dauererregten Zustand ausharren, in einem aktivierten Nervensystem, das gar nicht mehr richtig runterfährt.

Vielleicht gehören Sie zu den wenigen Glücklichen, die noch keine digitale Abhängigkeitsstruktur aufweisen. Die ihr Smartphone in eine Ecke schiessen können und es drei Tage vergessen. Oder gar keines haben. Dann gehören Sie jedoch zu einer Minderheit und müssen spätestens als Fachperson im psychologischen Bereich

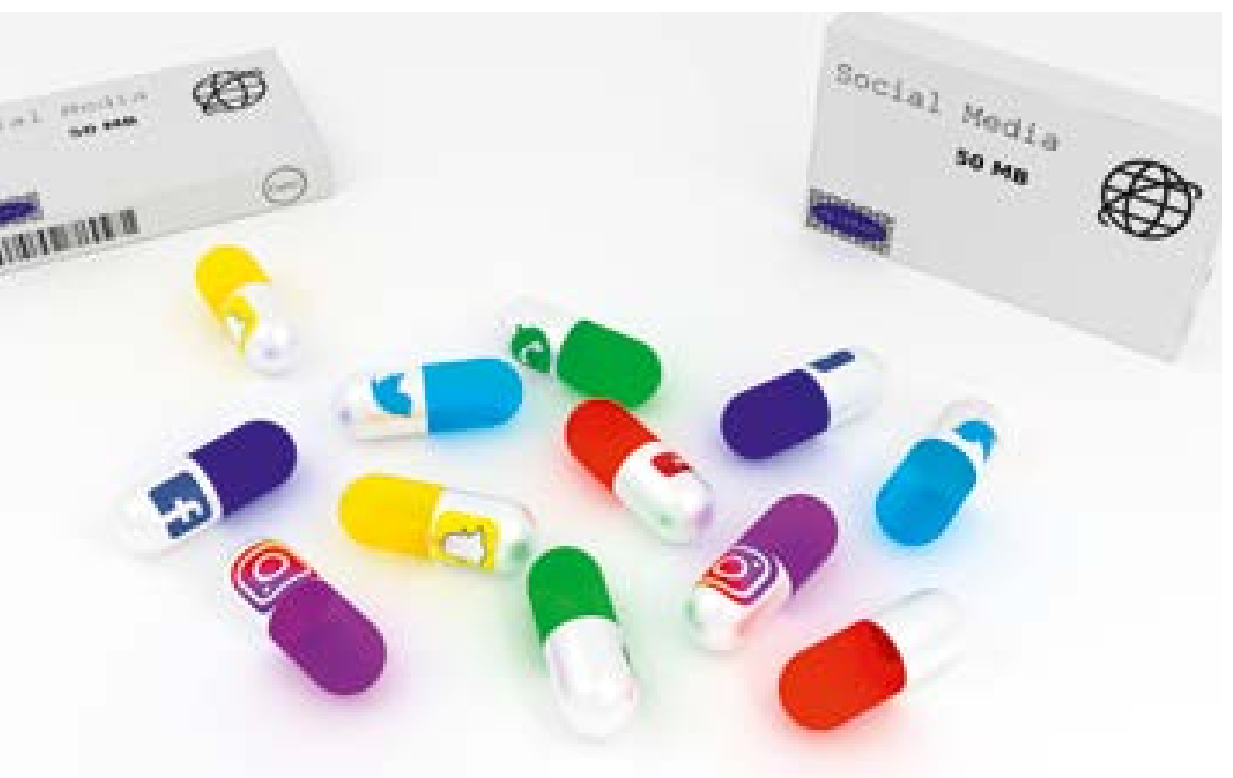

beginnen, zu verstehen, wie sehr digitale Möglichkeiten die Psyche und das Gehirn des Menschen seit Jahren verändern.

Was also tun? Das Erste ist, sich bewusst zu machen, dass die psychische Gesundheit in digitalen Zeiten ein grosses, komplexes und wichtiges Thema ist. Dass die Digitalisierung auf psychologischer und soziologischer Ebene massive Veränderungen mit sich bringt und wir uns Wissen aneignen müssen, um uns selbst, unsere Klient*innen und auch die Gesellschaft aufzuklären und adäquat begleiten zu können. Informieren Sie sich als Fachperson über digitale Abhängigkeitsstrukturen. Besuchen Sie eine Weiterbildung. Und sprechen Sie das Thema auch im therapeutischen Setting an.

Auch müssen wir selbst uns mit unserem digitalen Nutzungsverhalten auseinandersetzen. Ob Digitalisierung gut oder schlecht ist, ist nicht mehr die Frage. Sie ist da, sie ist allgegenwärtig, sie ist schlicht eine Tatsache, und sie birgt viele Chancen. Richtig und bewusst eingesetzt, ermöglicht uns die Digitalisierung ein besseres Leben. Doch wir müssen damit beginnen, sie nachhaltig einzusetzen. So, dass unsere Psyche davon profitieren kann, statt sich daran kaputt zu konsumieren.

Beginnen Sie gleich heute damit, für sich selbst in eine grössere digitale Balance zu kommen. Reflektieren Sie Ihr eigenes Nutzungsverhalten. Löschen Sie zeitfressende Apps, räumen Sie digital auf, kaufen Sie sich einen analogen Wecker, verbannen Sie das Smartphone aus dem Schlafzimmer. Planen Sie Konzentrationsinseln im Alltag ein, um sich effizienter auf wichtige Aufgaben konzentrieren zu können. Nehmen Sie Ihr Bedürfnis nach Ruhe und Verbundenheit ernst, indem Sie das Handy mal beiseitelegen und: im Hier und Jetzt ankommen.

Anna Miller ist Journalistin, Autorin, Positive Psychologin und Gründerin des Digital Balance Lab. Sie berät Private und Unternehmen zur Frage, wie wir glücklicher sein können in einer immer digitaleren Welt. www.anna-miller.ch www.digitalbalancelab.com 